\title{
«Dieser Verfassungsartikel ist ein richtungsweisender Systementscheid»
}

\author{
Peter Tschudi, Professor für Hausarztmedizin an der Universität Basel, ist einer der \\ «Väter» der Volksinitiative «Ja zur Hausarztmedizin», die zugunsten des direkten \\ Gegenentwurfs zurückgezogen wurde, über den am kommenden Wochenende ab- \\ gestimmt wird. Im folgenden Interview nimmt er zum Verfassungsartikel «Medizi- \\ nische Grundversorgung» Stellung.
}

Interview: Bruno Kesseli

bkesseli[at]emh.ch

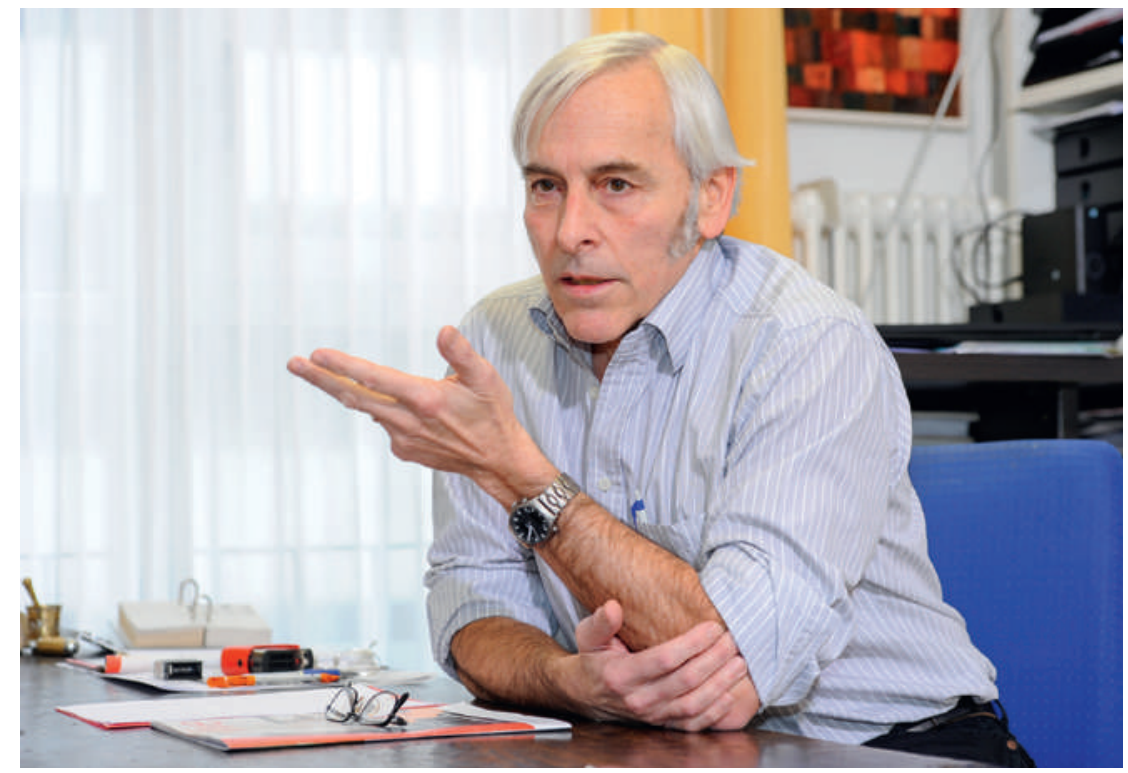

«Es ging uns nie um den «Heimatschutz» unseres Berufes, sondern um die medizinischen Grundversorgung der Bevölkerung auf lange Sicht»: Peter Tschudi zur Abstimmung über den Verfassungsartikel «Medizinische Grundversorgung».
Parallel zur Phase der parlamentarischen Beratung der Initiative haben wir in den letzten Jahren die Bevölkerung laufend über die besorgniserregende Entwicklung der Hausarzt- und Kindermedizin informiert, unter anderem mit einem Hausärzte-Bus, der seit 2011 durch die Schweiz tourte. Dies trägt nun Früchte. Die Bevölkerung hat erkannt, dass es um ihre Gesundheit, um ihren eigenen Haus- und Kinderarzt in ihrem Quartier/Dorf geht. Immer wieder haben wir erfahren, dass die Schweizer Bevölkerung, ob in der Stadt oder auf dem Lande, sich eine hausarzt- und kinderarztbasierte Grundversorgung wünscht. Von allen Vorlagen, über die wir am 18. Mai abstimmen, hat diejenige über die medizinische Grundversorgung wohl die direktesten Auswirkungen für alle Einwohnerinnen und Einwohner. Eine gute Hausarzt- und Kindermedizin für alle überall in der ganzen Schweiz ist extrem wichtig.

Können Sie die wichtigsten Verbesserungen, die sich durch den neuen Verfassungsartikel ergeben würden, nochmals kurz zusammenfassen?

Mit der Vorlage über die «Medizinische Grundversorgung» soll erstmals eine gesundheitspolitische Zielsetzung in die Bundesverfassung festgeschrieben werden. Ordnungspolitisch bedeutet dies: Dieser Verfassungsartikel ist ein richtungsweisender Systementscheid. Die Schweiz, das Schweizervolk stellt die Weichen zu einem Gesundheitssystem, welches auf einer starken Grundversorgung und einer starken Hausarzt- und Kindermedizin basiert.

Wie hängen der neue Verfassungsartikel und der Masterplan zusammen?

Der Masterplan von Bundesrat Alain Berset enthält alle wesentlichen Punkte für eine Besserstellung der Hausarzt- und Kindermedizin. Der neue Verfassungsartikel ist die Garantie dafür, dass der ausgehandelte Masterplan umgesetzt wird. Besonders wichtig ist dabei das Bekenntnis zu einem Gesund- 
heitssystem, das auf einer starken medizinischen Grundversorgung und somit auf einer starken Hausarzt- und Kindermedizin beruht. Die Festschreibung dieses Prinzips ist für uns das Wichtigste, weil es im Interesse aller ist. Und es ist ja nicht in erster Linie

\section{«Der neue Verfassungsartikel ist die Garantie dafür, dass der ausgehandelte Masterplan umgesetzt wird.»}

der Beruf des Haus- und Kinderarztes, der in der Verfassung verankert werden soll, sondern vielmehr seine Funktion. Das ist durchaus sinnvoll. Damit werden der Auftrag und die Bedeutung der medizinischen Grundversorgung und der Hausarzt- und Kindermedizin langfristig gesichert.

Die Stimmen aus der Ärzteschaft, die sagen, der Verfassungsartikel unterlaufe die ursprünglichen Anliegen der Initiative «Ja zur Hausarztmedizin» zumindest teilweise, liegen also falsch?

Ja, die liegen falsch, weil es keinen Sinn macht. Wieso sollten wir einem Verfassungsartikel zustimmen, der unsere eigene Initiative unterläuft? Dank der starken Verankerung der Hausarzt- und Kinder- sche Grundversorgung im Allgemeinen und die Stärkung der Hausarzt- und Kindermedizin im Besonderen geschaffen.

Hätte man nicht trotzdem an der Initiative festhalten sollen?

Die Anliegen der Initianten sind von den Behörden aufgegriffen und viele Forderungen rascher erfüllt worden, als dies bei einem erfolgreichen Ausgang einer Abstimmung über den ursprünglichen Initiativtext der Fall gewesen wäre. Insofern sind die eingeleiteten Massnahmen - u. a. der Masterplan - und der Gegenentwurf der Bundesbehörden zu begrüssen. Nachdem sich die Initianten, das Parlament, der Bundesrat und weitere Akteure im Gesundheitswesen auf ein gemeinsames Vorgehen und Programm geeinigt hatten, hat das Initiativkomitee Ende September 2013 deshalb seine Initiative zurückgezogen. Dies auch als Ausdruck unserer Kompromissbereitschaft, denn es ging uns nie um den «Heimatschutz» unseres Berufes, sondern um die medizinische Grundversorgung der Bevölkerung auf lange Sicht.

Was bedeutet es für die Hausarzt- und Kindermedizin in der Schweiz, wenn die Abstimmung gewonnen wird? Dass die Grundversorgung und die Hausarzt- und Kindermedizin für alle Menschen in der Schweiz definitiv erhalten bleiben. Zudem bedeutet es, dass die

\section{«Ich rechne mit einem klaren Sieg und der Zustimmung aller Kantone. Je höher, desto besser.»}

medizin in der Bevölkerung hat die Volksinitiative «Ja zur Hausarztmedizin» die Politik in Bewegung gebracht und Parlament und Regierung zum Handeln veranlasst! Inzwischen haben Bundesrat und Parlament nicht nur die wesentlichen Forderungen der Volksinitiative umgesetzt - Stärkung der Aus- und Weiterbildung, Erleichterungen in der Berufsausübung, z.B. im Laborbereich, Korrekturen im Tarifsystem -, sondern mit dem neuen Verfassungsartikel auch die nachhaltige Grundlage für die medizini-
Hausarzt- und Kindermedizin Einzug in die Bundesverfassung erhalten und damit als Dauerauftrag verfassungsrechtlich verankert werden.

Als einer der Väter der Hausarztinitiative rechnen Sie wohl mit einem Abstimmungserfolg. Mit welchem Stimmenverhältnis wären Sie glücklich?

Ich rechne mit einem klaren Sieg und der Zustimmung aller Kantone. Je höher, desto besser. Vielen Dank! 